\title{
X-ray-Activated Simultaneous Near-Infrared and Short-Wave Infrared Persistent Luminescence Imaging for Long-Term Tracking of Drug Delivery
}

Dandan Ding, ${ }^{\dagger}$ Shi Li,,$^{\dagger}$ Han Xu,,$^{\dagger}$ Luchao Zhu, ${ }^{+}$Shanshan Meng, ${ }^{\dagger}$ Jun Liu, ${ }^{+}$Qin Lin,,${ }^{+}$ Stephen Wan Leung, ${ }^{\#}$ Wenjing Sun, ${ }^{*}+{ }^{\dagger}$ Yimin Li, ${ }^{*}, \dot{+}$ Hongmin Chen ${ }^{* \dagger}$

$\uparrow$ State Key Laboratory of Molecular Vaccinology and Molecular Diagnostics \& Center for Molecular Imaging and Translational Medicine, School of Public Health, Xiamen University, Xiamen 361102, China

+ Department of Radiation Oncology, Cancer Center, the First Affiliated Hospital of Xiamen University, Xiamen 361003, China

\# Department of Radiation Oncology, Kaohsiung Yuan's General Hospital, Kaohsiung, Taiwan

Corresponding Author

* E-mail: sunwenjing1102@163.com

*E-mail: lym200528@126.com

* E-mail: hchen@xmu.edu.cn 


\section{Materials}

Magnesium nitrate hexahydrate $\left(\mathrm{Mg}\left(\mathrm{NO}_{3}\right)_{2} \cdot 6 \mathrm{H}_{2} \mathrm{O}, 99 \%\right)$, ytterbium nitrate hexahydrate $\left(\mathrm{Yb}\left(\mathrm{NO}_{3}\right)_{3} \cdot 6 \mathrm{H}_{2} \mathrm{O}, 99.9 \%\right)$, tetraethyl orthosilicate (TEOS, 99\%), cetyltrimethylammonium bromide (CTAB, 99\%), and 4-nitrophenyl chloroformate 3-(4,5-dimethylthiazol-2-yl)-2,5-diphenyltetrazolium bromide (MTT) were purchased from Sigma-Aldrich. Germanium dioxide $\left(\mathrm{GeO}_{2}, 99 \%\right)$ and doxorubicin hydrochloride $(\mathrm{DOX} \cdot \mathrm{HCl})$ were purchased from Aladdin Reagents. Ammonia aqueous solution $(28 \mathrm{wt} \%)$ and ethanol were supplied by Sinopharm Chemical Reagent Co., Ltd. Deionized water $\left(18.2 \mathrm{M} \Omega \mathrm{cm}^{-1}\right)$ was obtained from a Milli-Q ultrapure system. Fetal bovine serum (FBS) was purchased from HyClone Laboratories Inc. All chemicals were used without further purification unless noted. HepG2 cells were obtained from Institute of Biochemistry and Cell Biology (Shanghai, China).

\section{Characterization}

Crystal structure and phase composition were determined by X-ray diffraction (XRD) measurement using an X-ray powder diffractometer (Bruker, D8 ADVANCE) with $\mathrm{Cu}$ K $\alpha$ radiation $(\lambda=1.5406 \AA)$ at $40 \mathrm{kV}$ and $40 \mathrm{~mA}$. Transmission electron microscopy (TEM) images were obtained using a microscope at an acceleration voltage of $200 \mathrm{kV}$ (FEI Tecnai G2 F30). X-ray excited NIR persistent luminescence imaging was conducted on an IVIS spectrum in vivo imaging system (Caliper IVIS, Lumina II). X-ray excited SWIR persistent luminescence was conducted on NIR-OPTICS Series III 900/1700 and In-Vivo Master. X-ray excited optical luminescence (XEOL) and 
X-ray excited persistent luminescence (XEPL) spectra were measured using an Ocean Optics QE Pro optical bench. Persistent luminescence decay curves were acquired by FLS980 Series of Fluorescence Spectrometers. The mini-X X-ray tube (Amptek Inc.) was set at $50 \mathrm{kV} / 70 \mu \mathrm{A}$. The fluorescence images of cells were taken on a laser scanning confocal microscopy (Olympus FV1200, Japan).

\section{Synthesis of MGO}

MGO nanoparticles were prepared through hydrothermal reaction and annealing treatment. Firstly, $\mathrm{GeO}_{2}$ was dissolved in $\mathrm{NaOH}(2 \mathrm{M})$ aqueous solution. Then 1 mmol $\mathrm{Mg}^{2+}, 1.25 \mathrm{mmol} \mathrm{Ge}^{4+}$ and $0.001 \mathrm{mmol} \mathrm{Yb}^{3+}$ were dissolved in $25 \mathrm{~mL}$ of deionized water under vigorous stirring, and ammonium hydroxide (28 wt \%) was then added to adjust the $\mathrm{pH}$ value to $\sim 8$. The mixture was vigorously stirred for $2 \mathrm{~h}$, and transferred into a $50 \mathrm{~mL}$ Teflon-lined autoclave and reacted at $200{ }^{\circ} \mathrm{C}$ for $12 \mathrm{~h}$. After cooling to room temperature (r.t.), the precipitates were collected by centrifugation and washed for three times with deionized water. The collected precipitates were dried in vacuum at $60{ }^{\circ} \mathrm{C}$ and annealed at $1100{ }^{\circ} \mathrm{C}$ in air for $2 \mathrm{~h}$ to obtain persistent luminescent property. For further bio-applications, the MGO powder was wet ground in $\mathrm{NaOH}(5 \mathrm{mM})$ solution and re-dispersed in $\mathrm{NaOH}(5 \mathrm{mM})$ solution by sonication for $1 \mathrm{~h}$. The resulting solution was centrifuged at $3000 \mathrm{rpm}$ for $10 \mathrm{~min}$ to remove large size particles, and the supernatant was re-centrifuged at $10000 \mathrm{rpm}$ for 5 min to collect the MGO nanoparticles.

\section{Loading of DOX}

mMGOs (1 mg) and DOX (1 mg) were dispersed in $2 \mathrm{~mL}$ ultrapure water and the 
mixture was vigorously stirred for $24 \mathrm{~h}$ at r.t. The obtained DOX-loaded mMGOs (DOX-mMGOs) nanoparticles were centrifuged at $10000 \mathrm{rpm}$ for $5 \mathrm{~min}$ and washed for three times with ultrapure water. The DOX loading content (LC) was calculated according to the following equation:

$$
\mathrm{LC}_{D O X}=\frac{W_{\text {DOX }}}{W_{\text {mMGOs }}} \times 100 \%
$$

$W_{\text {DOx }}$ : the loading weight of DOX, $W_{\text {mMGOs }}$ : the mass of the DOX-mMGOs.

\section{Drug release}

In order to evaluate the DOX release behavior, DOX-mMGOs $(2 \mathrm{mg})$ were dispersed in $2 \mathrm{~mL}$ of PBS solution with different $\mathrm{pH}$ values $(5.6$ and 7.4$)$ at $37{ }^{\circ} \mathrm{C}$ under continuous shaking. At 1, 2, 4, 8, 12, 24, and $48 \mathrm{~h}$, the samples were centrifuged, and the absorbance at $480 \mathrm{~nm}$ of the supernatant was measured using UV-Vis-NIR spectrophotometer. The release percentage (RP) was evaluated from the equation:

$$
\mathrm{RP}=\frac{W_{\text {released Dox }}}{W_{\text {DOX }}} \times 100 \%
$$

$W_{\text {released DOX }}$ : the DOX weight in supernatant, $W_{\text {DOX }}$ : the total loading weight of DOX in DOX-mMGOs.

\section{Animal experiments}

Female BALB/c nude mice (18-22 g) were purchased from Shanghai SLAC Laboratory Animal Co. Ltd (Shanghai, China), and all of the in vivo experiments were carried out according to the criteria of the National Regulation of China for Care and Use of Laboratory Animals.

In vivo low dose of X-ray recharged deep tissue NIR persistent luminescence imaging by intramuscular injection 
The in vivo deep tissue imaging was examined by intramuscular injection of mMGOs solution $\left(200 \mu \mathrm{L}, 1 \mathrm{mg} \mathrm{mL}^{-1}\right)$ into the right leg of normal nude mice. $1.5 \mathrm{~cm}$ thick of pork tissue was placed between the hind leg of normal mouse and X-ray source. The images were recorded immediately after low dose of X-ray irradiation ( $25 \mathrm{mGy})$, with the pork remained on top. After $10 \mathrm{~min}$, the intramuscular injection site was recharged and the images were re-taken. The same process was repeated for three times totally.

\section{In vivo blood circulation and biodistribution}

To evaluate the in vivo biodistribution of mMGOs, tumor-bearing mice were established by subcutaneously injecting $5 \times 10^{6}$ HepG2 cells in the left flank of each BALB/c mouse and intravenously injected with mMGOs $\left(3.5 \mathrm{mg} \mathrm{kg}^{-1}\right)$. The main organs including heart, liver, spleen, lung, kidney, tumors, and blood were collected at various time points $(1,2,4,8,12,24$, and $48 \mathrm{~h})$ post-injection and were weighed and digested using $\mathrm{HNO}_{3}-\mathrm{H}_{2} \mathrm{O}_{2}$ mixture. The $\mathrm{Mg}$ content in all samples was measured by ICP-MS.

\section{Serum chemistry and hematology cell analysis}

BALB/c mice were intravenously injected with mMGOs $\left(3.5 \mathrm{mg} \mathrm{kg}^{-1}\right)$. Blood samples were collected for serum chemistry and blood cell analysis at day 0,3 , and 7 post-injection. Alanine transaminase (ALT), aspartate transaminase (AST), blood urea nitrogen (UREA) in serum were measured using an Automatic Biochemistry Analyzer (Mindray, BS-220). White blood cell (WBC), hemoglobin (HGB), red blood cell (RBC), hematocrit (HCT), mean corpuscular volume (MCV), mean corpuscular hemoglobin $(\mathrm{MCH}), \mathrm{RBC}$ distribution width (RDW-CV and RDW-SD), platelets 
(PLT), mean platelet volume (MPV), platelet distribution width (PDW) were measured using an Automatic Tri-classification Blood Cell Analyzer (Mindray, BC-2600). 


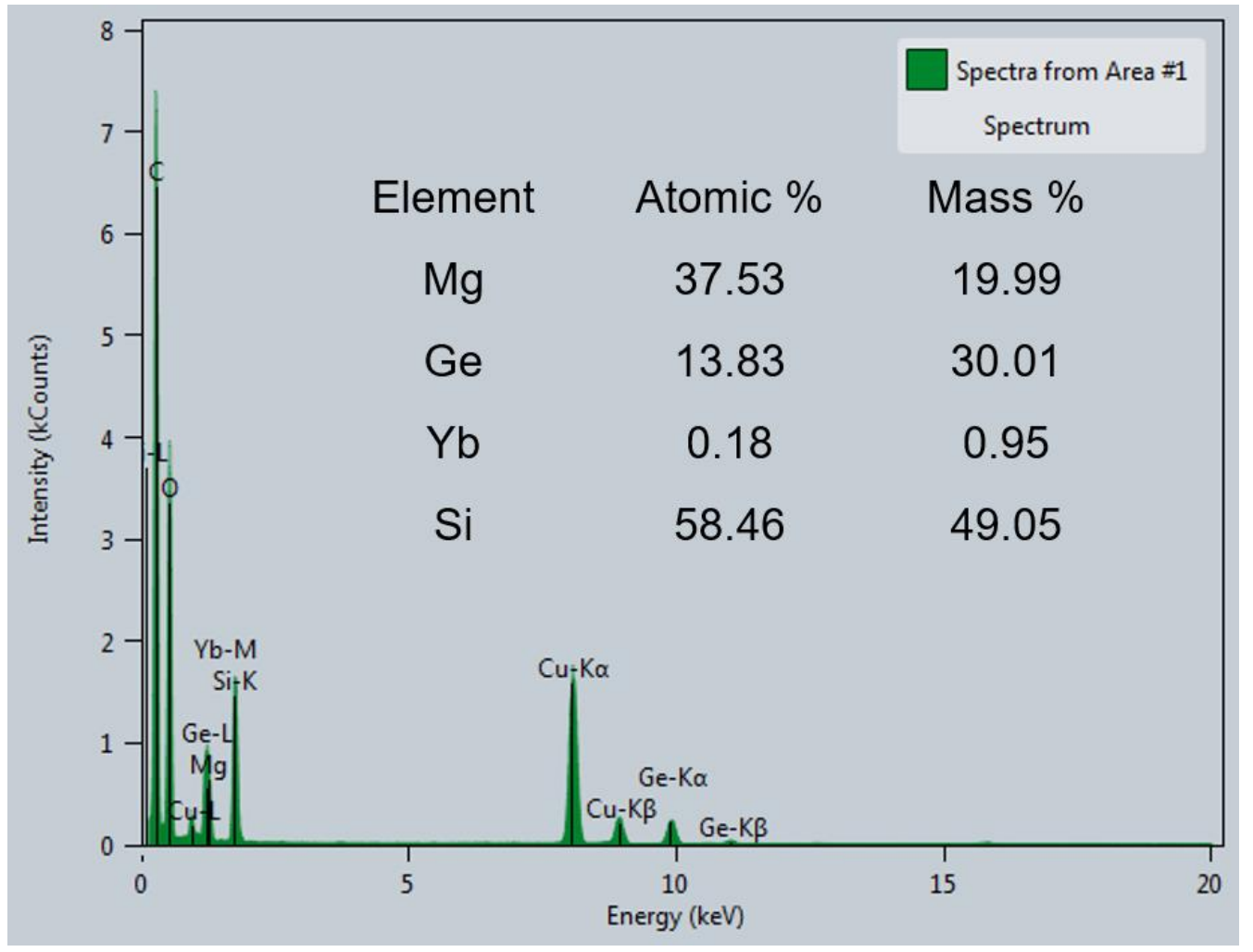

Figure S1. TEM-EDS result of the as-prepared mMGOs. Inset table showed the relative element contents. 


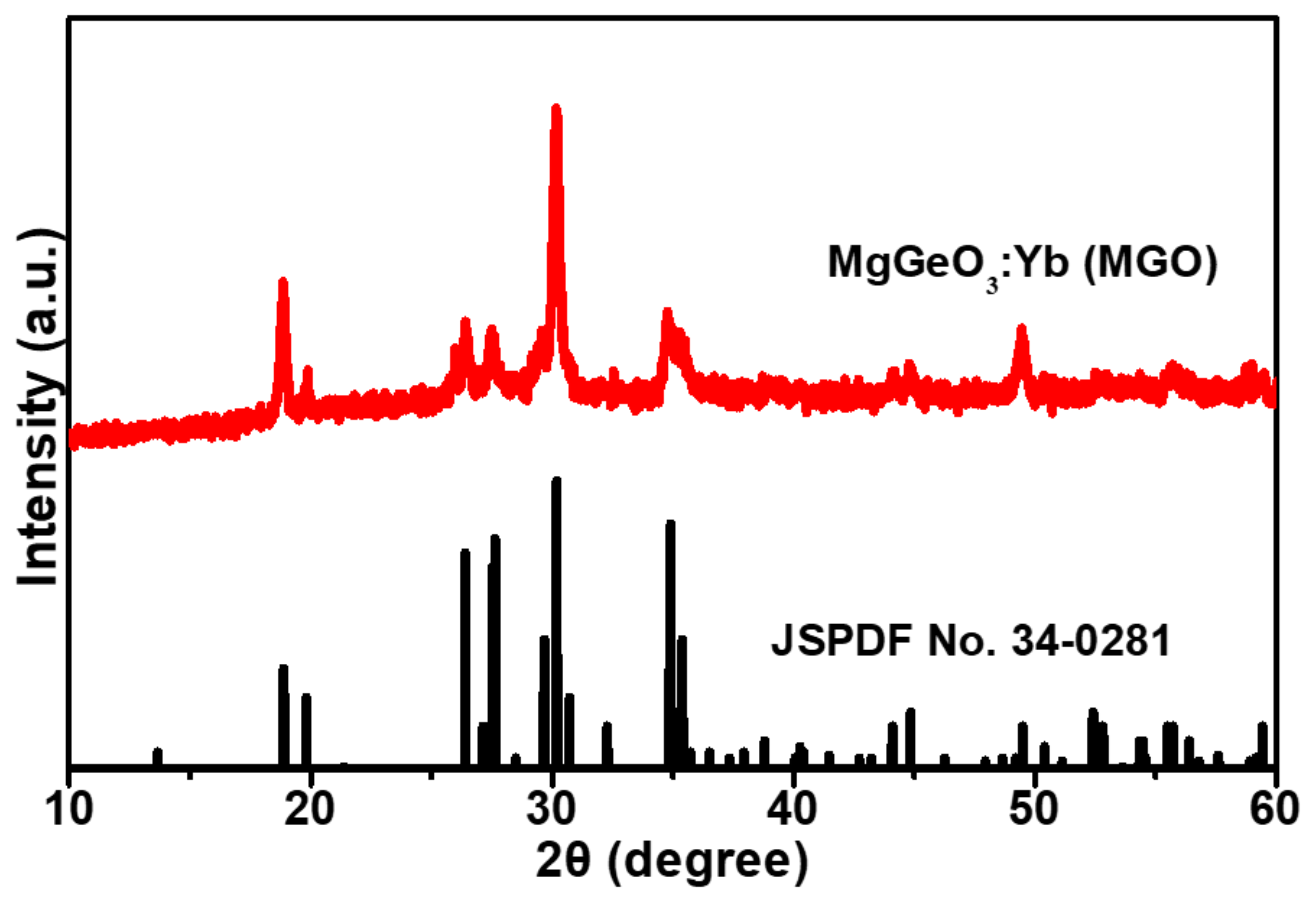

Figure S2. XRD analysis of MGO nanoparticles. 


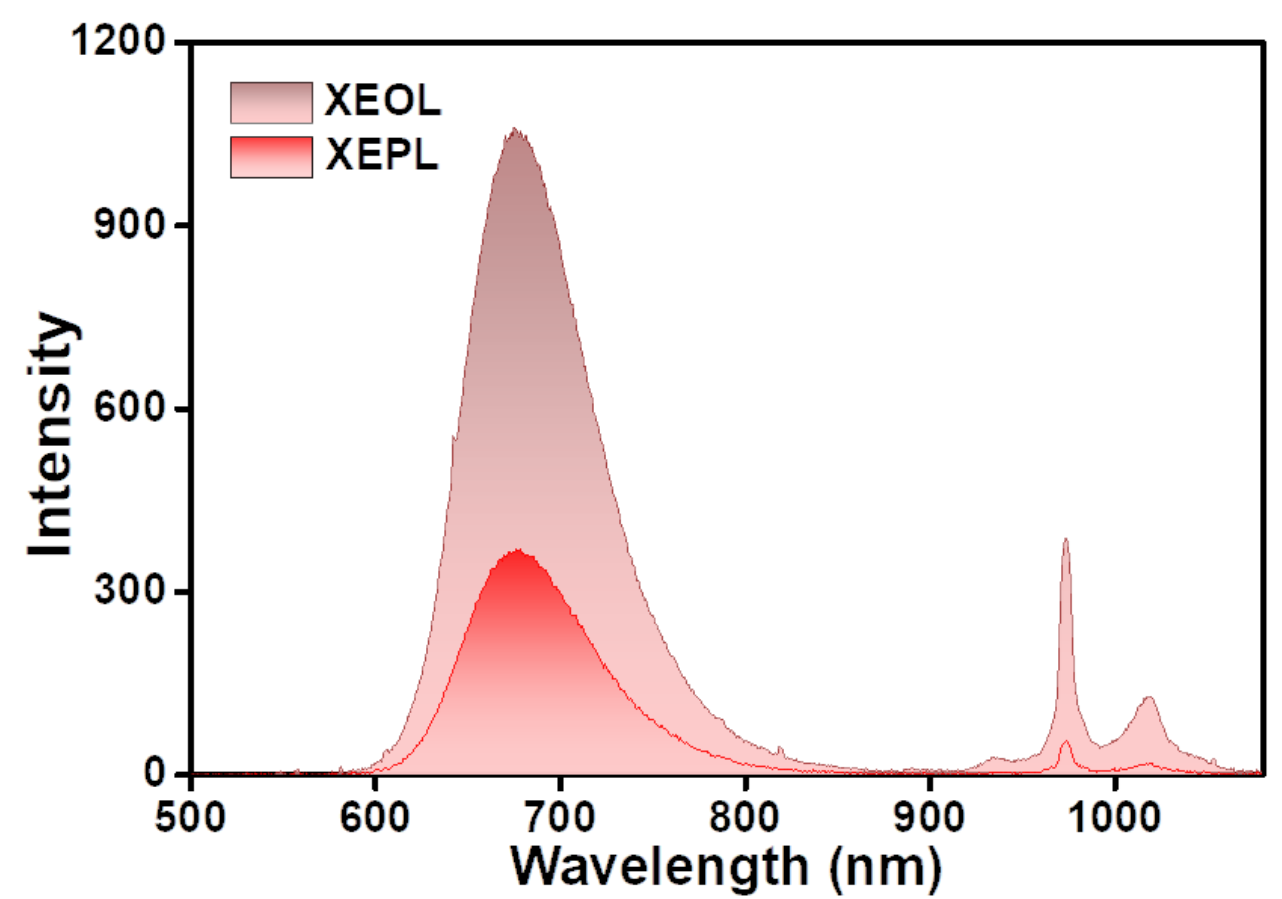

Figure S3. XEOL and XEPL spectra of MGO nanoparticles. 


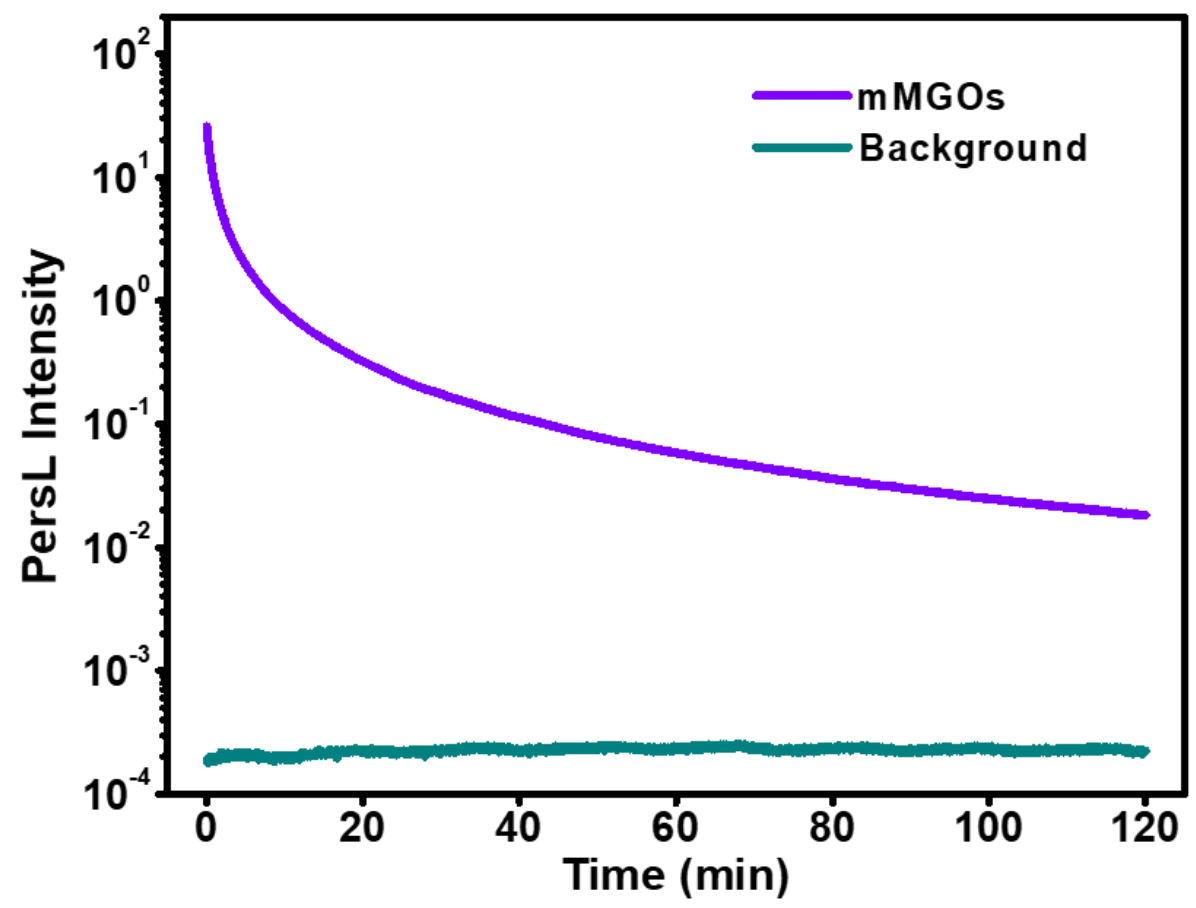

Figure S4. X-ray excited NIR persistent luminescence decay curve of mMGOs monitored at $670 \mathrm{~nm}$ after X-ray irradiation. 


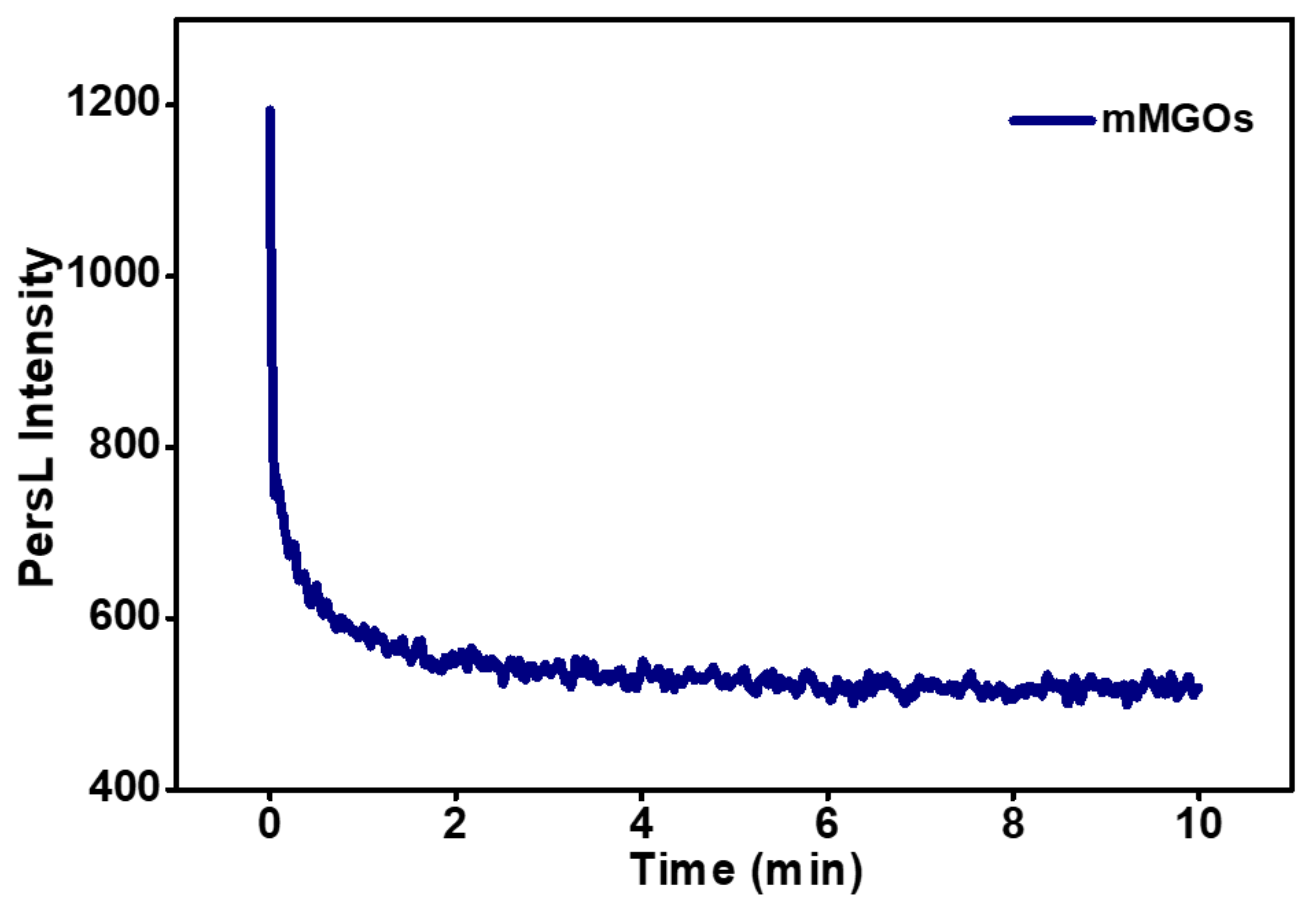

Figure S5. X-ray excited SWIR persistent luminescence decay curve of mMGOs monitored at $1019 \mathrm{~nm}$ after X-ray irradiation. 

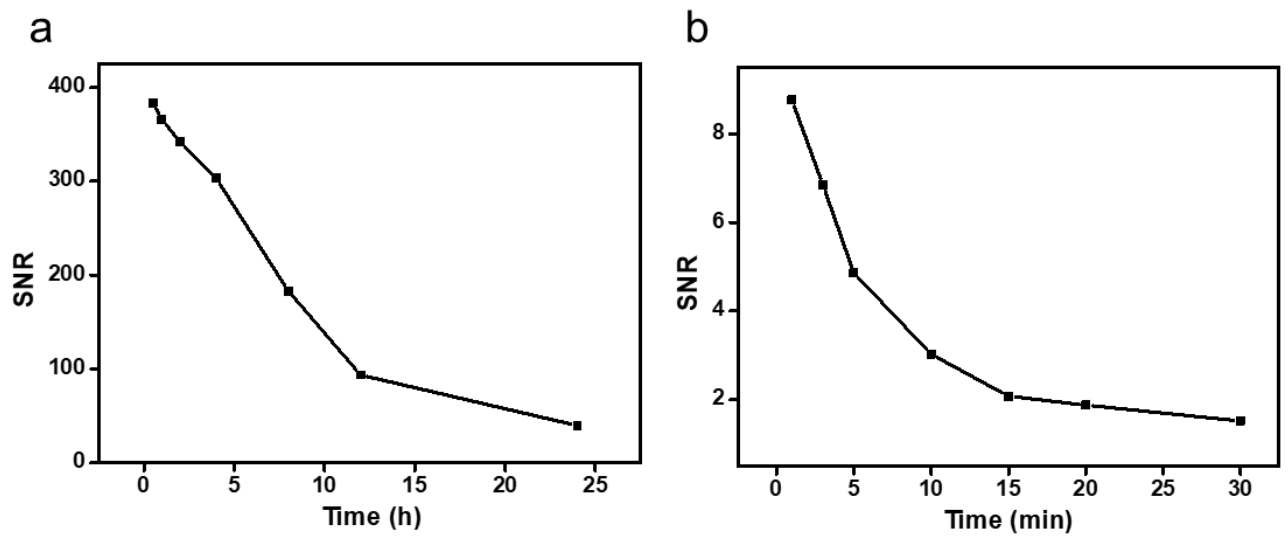

Figure S6. (a) SNR of NIR from Figure 1e. (b) SNR of SWIR from Figure 1f. 


\section{a}

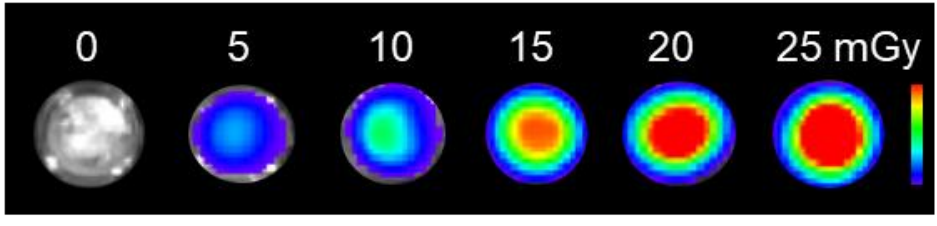

b

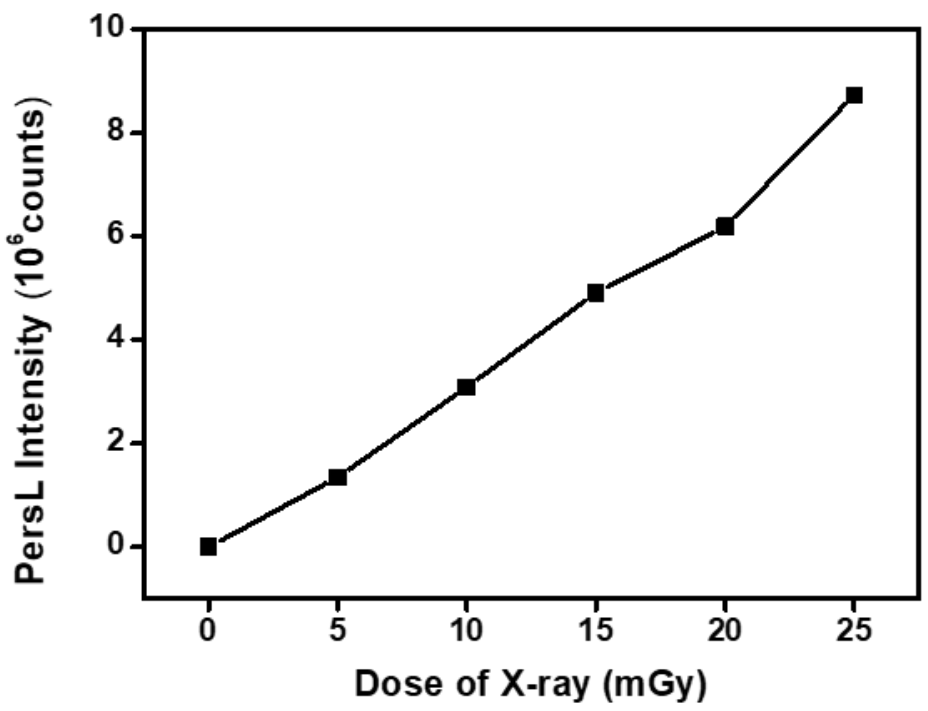

Figure S7. (a) NIR persistent luminescence decay images of the mMGOs after $1 \mathrm{~min}$ of X-ray irradiation with different dose. (b) The relationship between afterglow signal and the dosage of X-ray from (a) (Exposure time: 2s). 
a

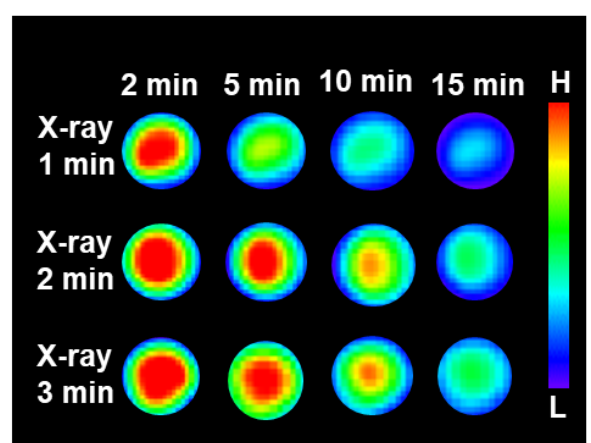

b

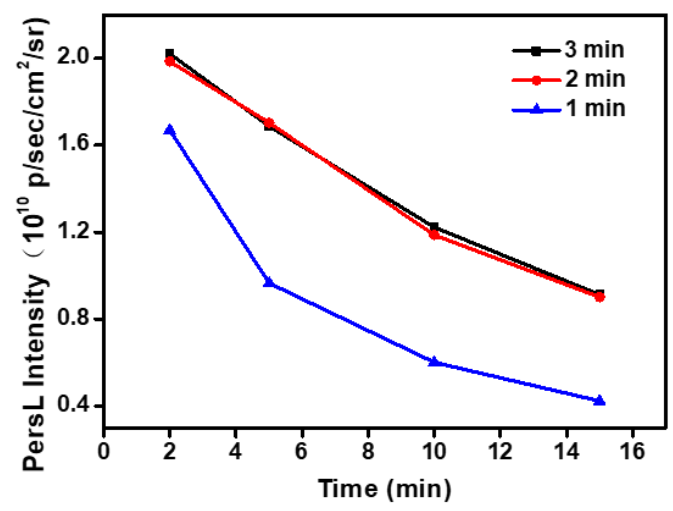

Figure S8. (a) NIR persistent luminescence decay images of the mMGOs after X-ray irradiation with different time. (b) Persistent luminescence intensity from (a) (Exposure time: $2 \mathrm{~s}$ ). 

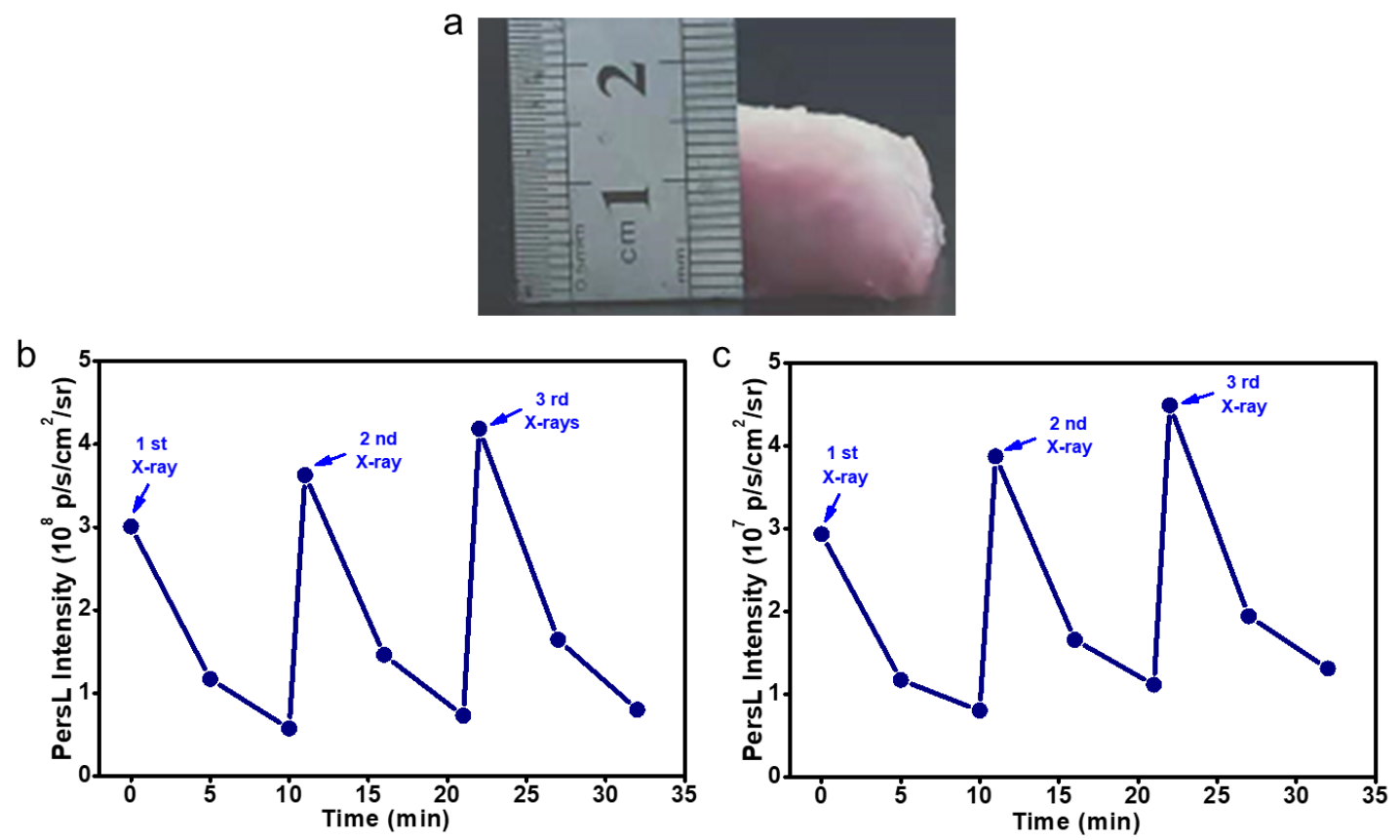

Figure S9. (a) The photo of $1.5 \mathrm{~cm}$ pork. (b) In vitro X-ray excited NIR persistent luminescence intensity changes based on the BLI imaging. (c) In vivo X-ray excited NIR persistent luminescence intensity changes based on the BLI imaging. 


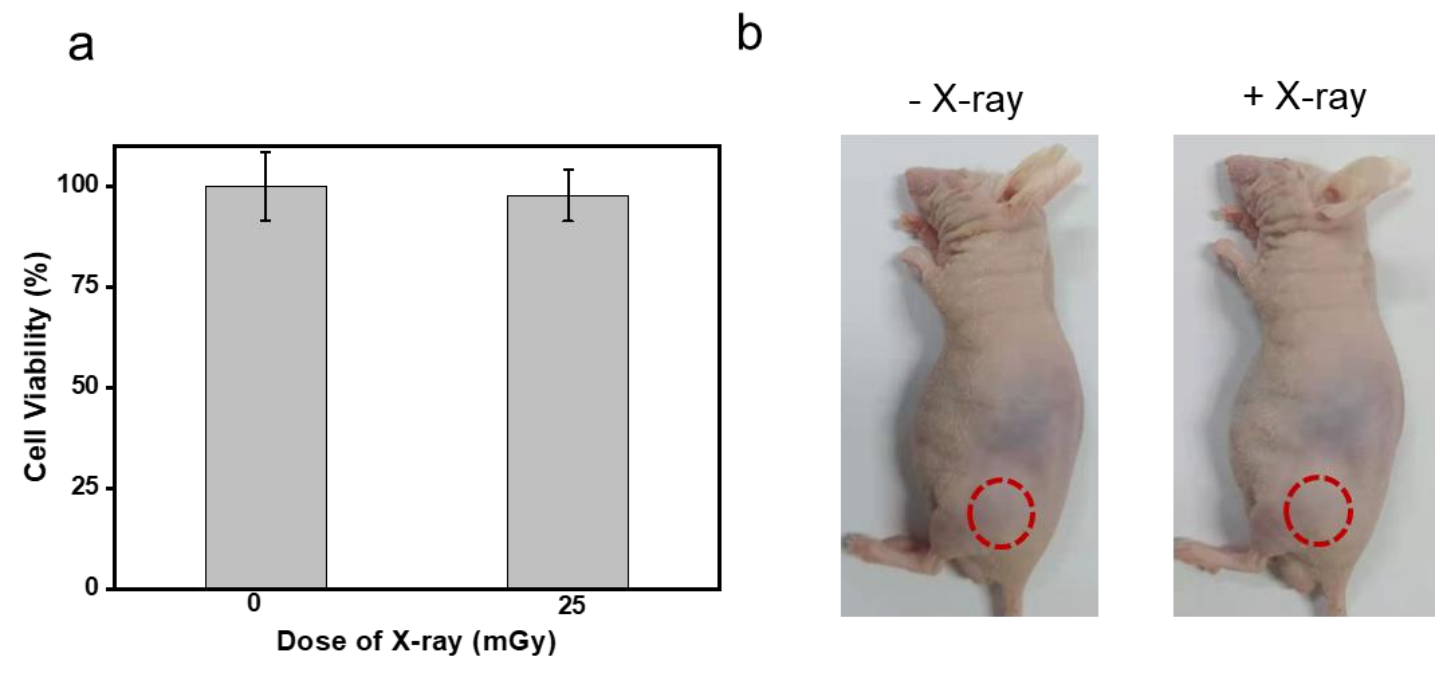

Figure S10. (a) Viability of HepG2 cells after $24 \mathrm{~h}$ of incubation with X-ray irradiation (0 and $25 \mathrm{mGy}$ ). (b) Photos of the mice before and after X-ray irradiation (25 mGy) (the red circle: irradiation site). 


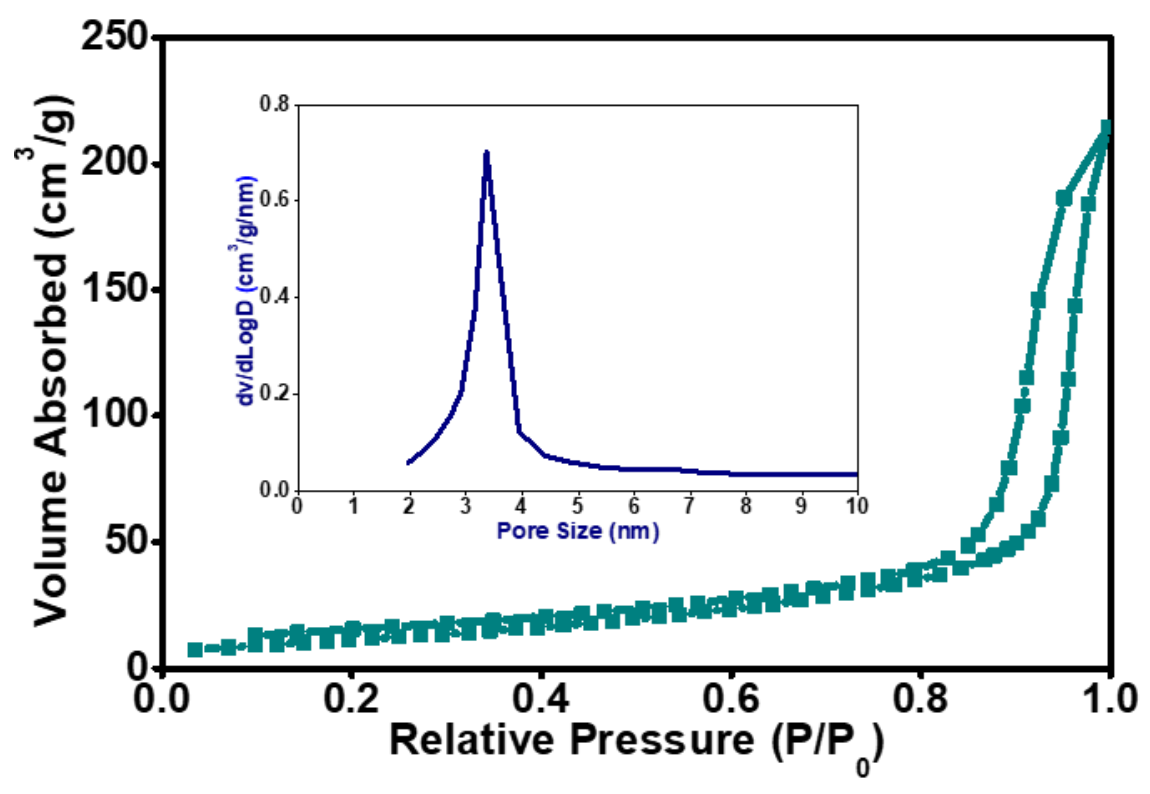

Figure S11. $\mathrm{N}_{2}$ adsorption/desorption isotherms and pore size distribution curves of mMGOs. 


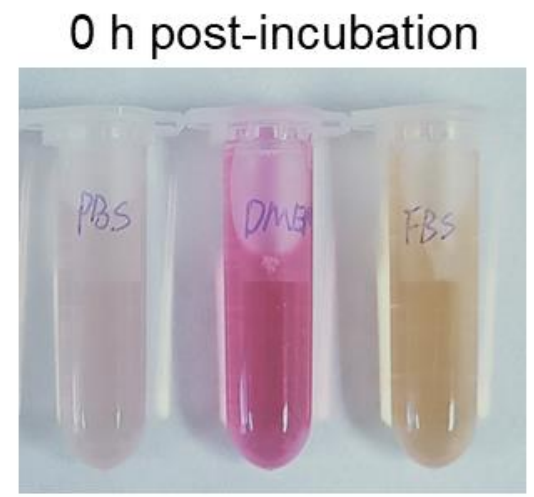

24 h post-incubation

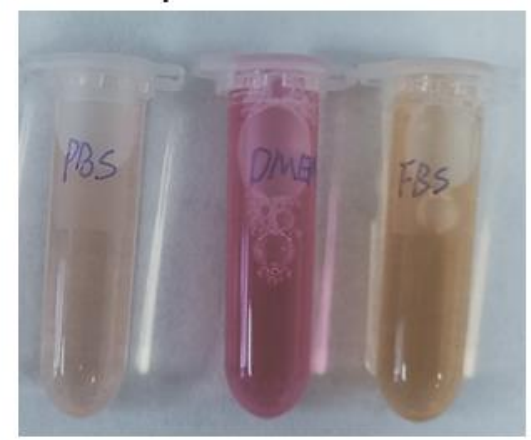

Figure S12. Photographs of DOX-mMGOs dispersed in PBS, DMEM, and FBS. 

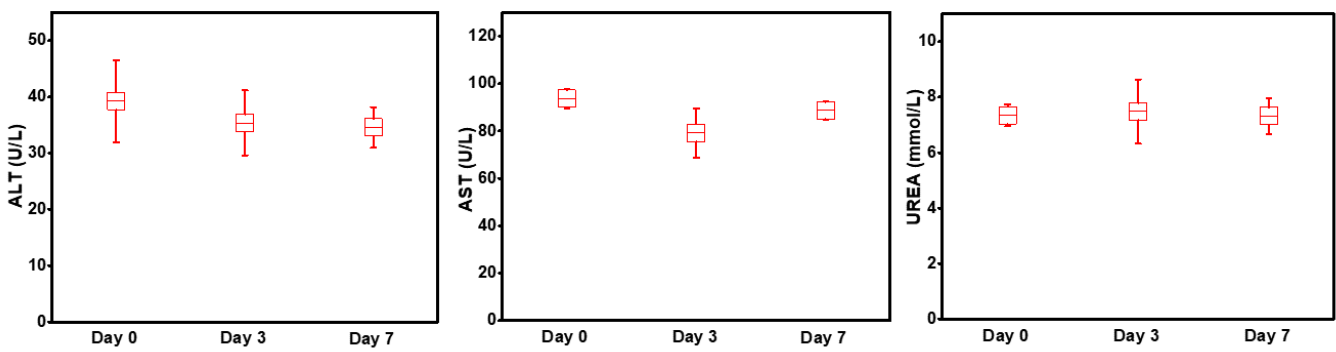

Figure S13. Serum chemistry of mice treated with PBS or DOX-mMGOs. ALT, alanine transaminase; AST, aspartate transaminase; UREA, blood urea nitrogen. Blood samples were collected for serum chemistry analysis on day 0 , day 3 , and day 7 after intravenous injection. 

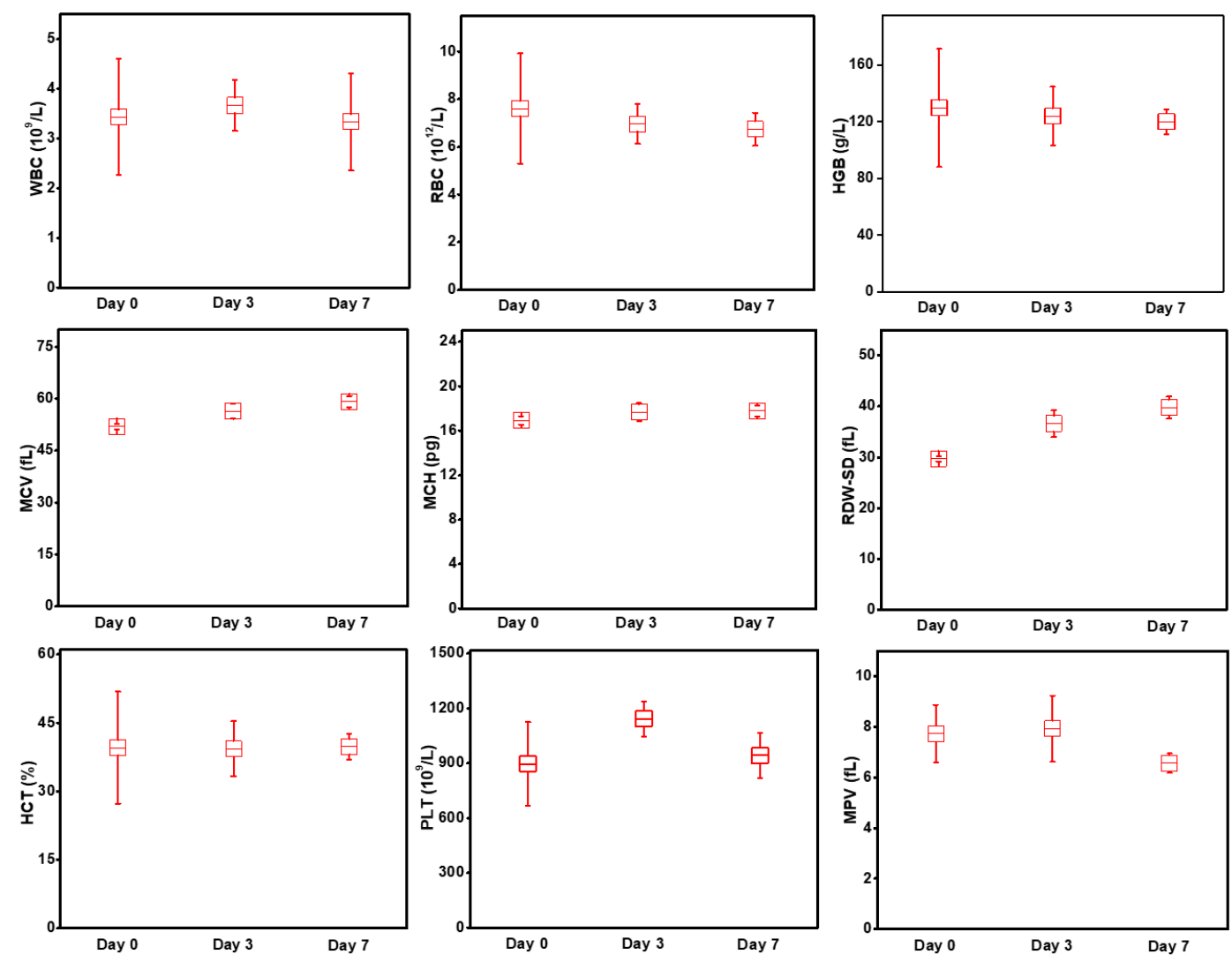

Figure S14. Hematological analysis of mice treated with PBS or DOX-mMGOs nanoparticles. WBC, white blood cell; RBC, red blood cell; HGB, hemoglobin; MCV, mean corpuscular volume; $\mathrm{MCH}$, mean corpuscular hemoglobin; RDW-SD, standard deviation of RBC distribution width; HCT, hematocrit; PLT, platelets; MPV, mean platelet volume. Blood samples were collected for hematological analysis on day 0 , day 3 , and day 7 after intravenous injection. 


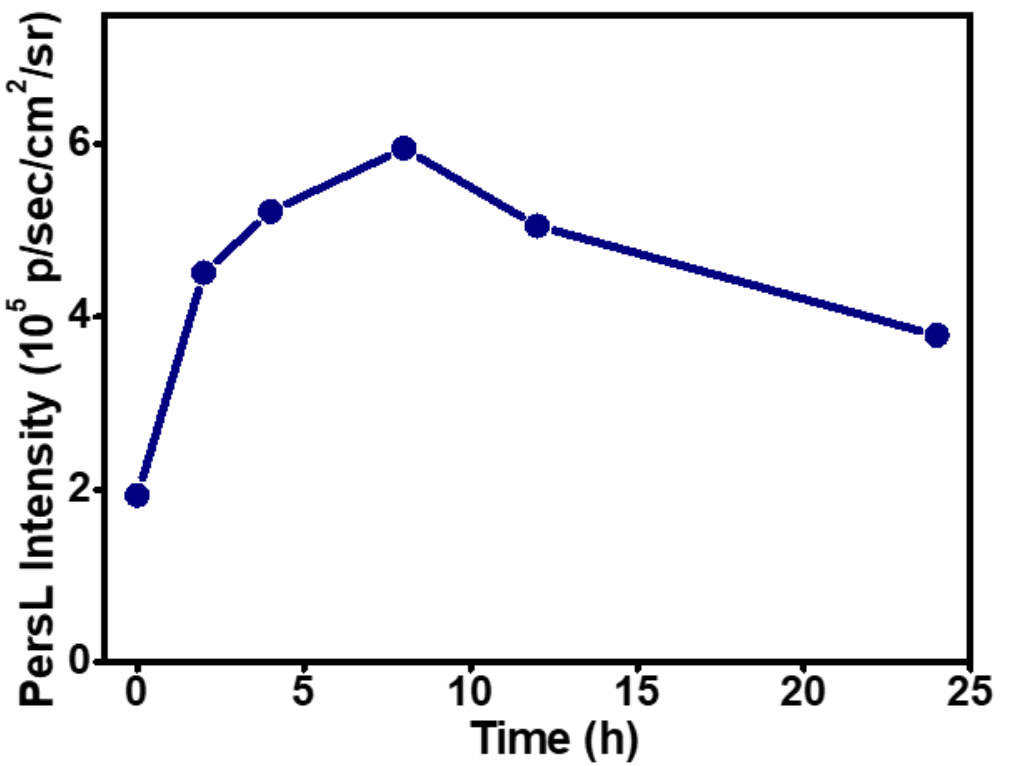

Figure S15. X-ray excited NIR persistent luminescence (PersL) intensity in tumors at different time points after intravenous injection. 


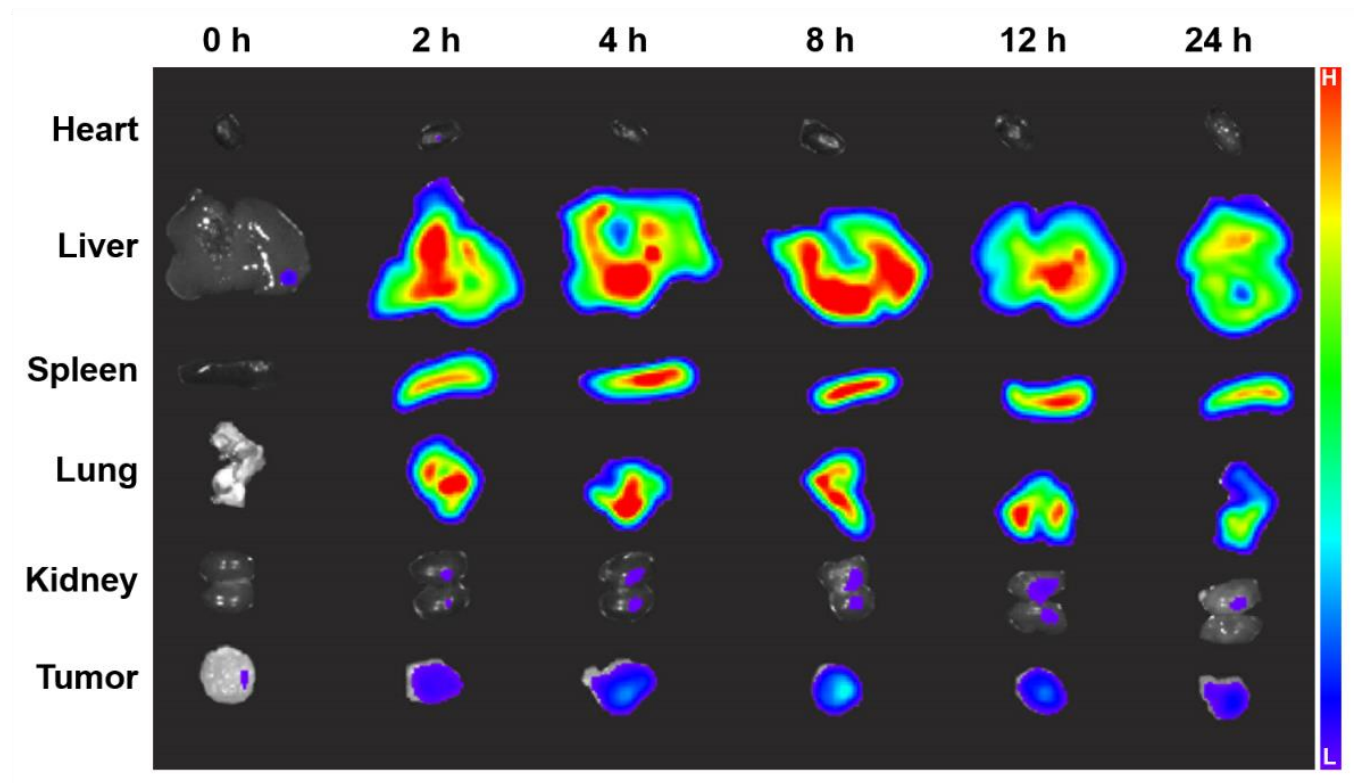

Figure S16. Ex vivo X-ray excited NIR persistent luminescence images of major organs at different time points after intravenous injection of mMGOs. 
a

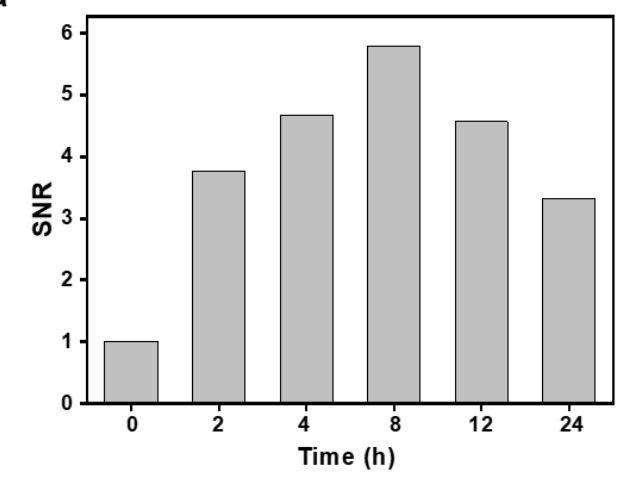

b

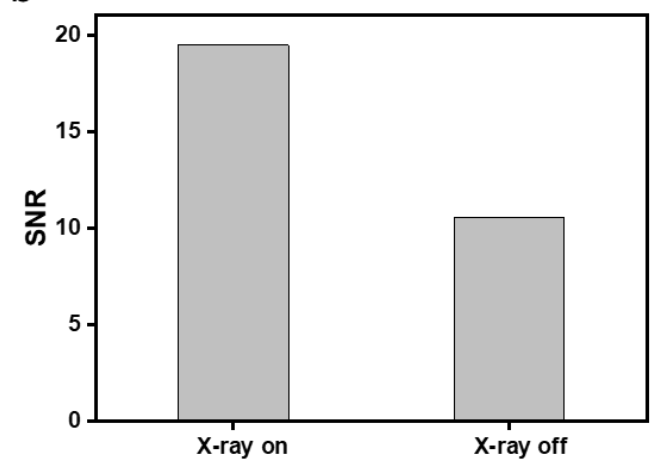

Figure S17. (a) SNR results from Figure 3c. (b) SNR results from Figure 3d. 


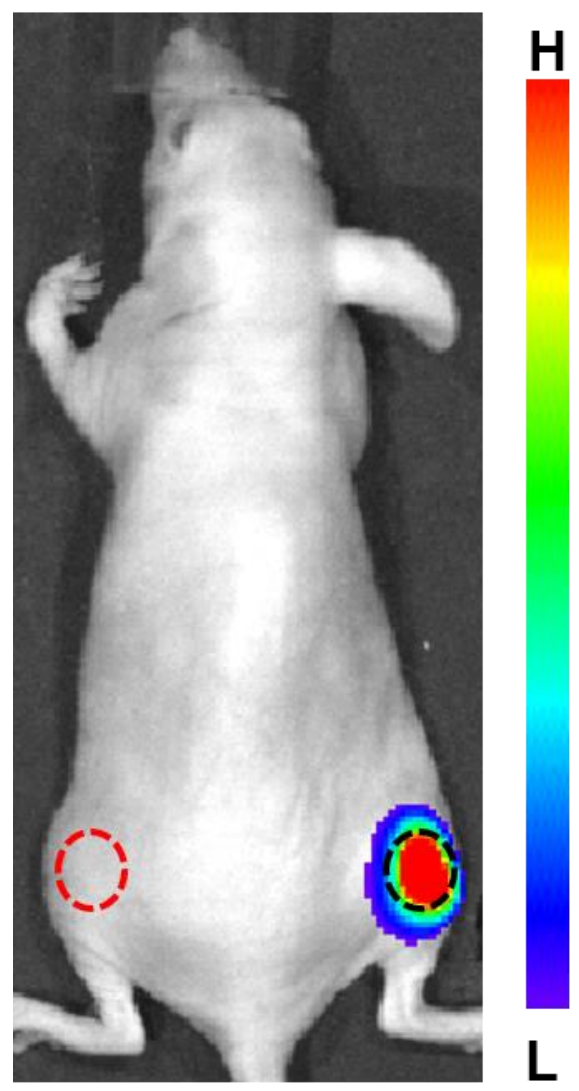

Figure S18. Persistent luminescence images of a mouse after intramuscular injection of mMGOs (the black circle: signal, the red circle: background). 


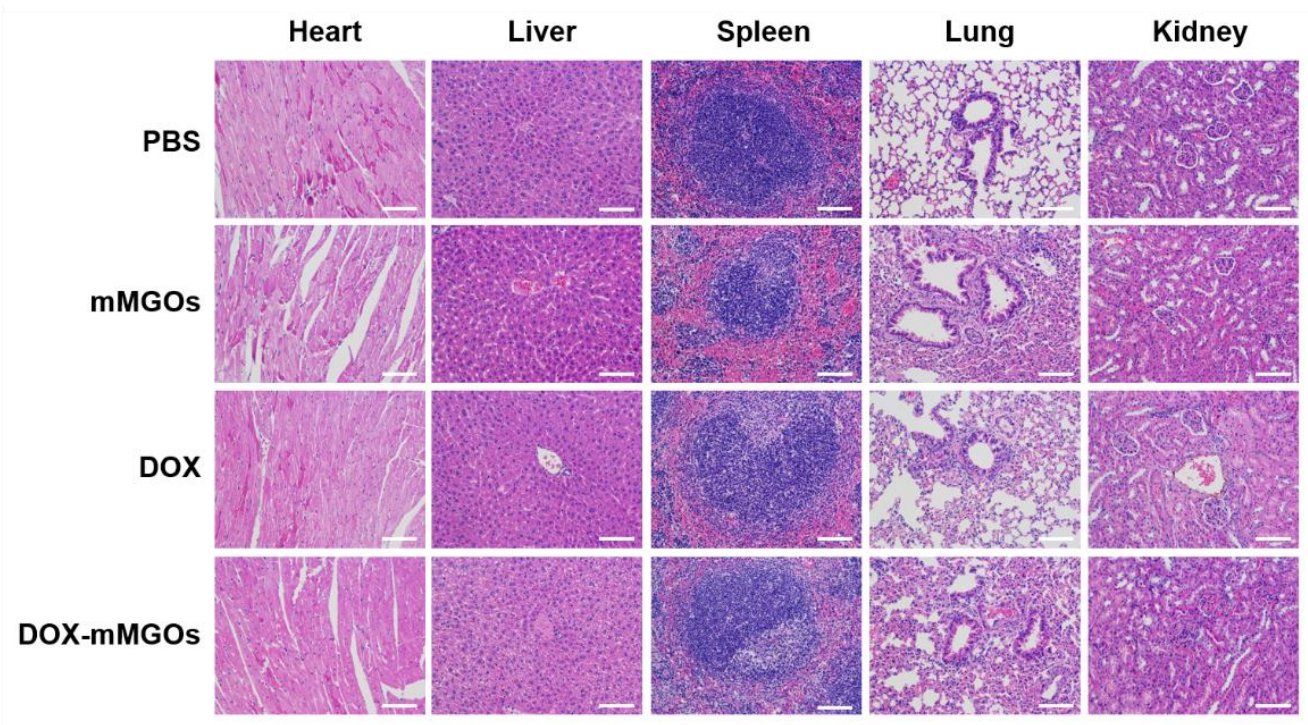

Figure S19. Hematoxylin \& eosin (H\&E)-stained slice images of major organs collected from different groups after 14 days of treatment. (Scale bar: $100 \mu \mathrm{m}$ ) 